\title{
Normal Subgroups of the Modular Group*
}

\author{
Leon Greenberg** and Morris Newman \\ Institute for Basic Standards, National Bureau of Standards, \\ Washington, D.C. 20234
}

(July 2, 1969)

A number of results on the normal subgroup structure of the classical modular group is announced. A typical result is that a normal subgroup of square-free index is necessarily of genus 1 , apart from 4 exceptions.

Key words: Genus; index; modular group; normal subgroups.

\section{Introduction}

In this note we summarize the results of some work on the normal subgroups of the classical modular group $\Gamma$, which is a continuation of the work begun in [1] ${ }^{1}$ and [4]. We may regard $\Gamma$ as the free product of a cyclic group of order 2 and a cyclic group of order $3 ; \Gamma=\{x\} *\{y\}, x^{2}=y^{3}=1$. The number of normal subgroups of $\Gamma$ of index $\mu$ will be denoted by $N(\mu)$. If $G$ is any subgroup of $\Gamma, G^{\prime}$ will denote its commutator subgroup, and $G^{p}$ the fully invariant subgroup of $G$ generated by the $p$ th powers of the elements of $G$. The level of $G$ is the least positive integer $n$ such that $(x y)^{n} \epsilon G$. If $G$ is a normal subgroup of index $\mu \geqslant 6$ and $n$ is its level, then the genus of $G$ is given by

$$
g=1+\mu(n-6) / 12 n,
$$

and the number of parabolic classes of $G$ by

$$
t=\mu / n \text {. }
$$

Except for the groups $\Gamma, \Gamma^{2}$, or $\Gamma^{3}$, the index of a normal subgroup is a multiple of 6 .

The commutator subgroup $\Gamma^{\prime}$ of $\Gamma$ is a free group of rank 2, freely generated by

$$
a=x y x y^{2}, \quad b=x y^{2} x y .
$$

The normal subgroups of $\Gamma$ of genus 1 (alternatively, of level 6) have been completely described in [5]. Any such subgroup $G$ lies between $\Gamma^{\prime}$ and $\Gamma^{\prime \prime}$ and may be described uniquely by the triplet of integers $(p, m, d)$, where $p>0,0 \leqslant m \leqslant d-1, m^{2}+m+1 \equiv 0 \bmod d . G$ is of index $6 d p^{2}$ in $\Gamma$ and consists of all words $w$ of $\Gamma^{\prime}$ satisfying

$$
e_{a}(w) \equiv 0 \bmod p, \quad e_{b}(w) \equiv m e_{a}(w) \bmod d p,
$$

where $e_{a}(w), e_{b}(w)$ are the respective exponent sums in $a$ and $b$ of $w$.

We also let $G_{k, m}$ be the intersection of all normal subgroups of $\Gamma$ containing

$$
(x y)^{m k}, \quad(y x)^{k}(x y)^{-k} .
$$

*This paper was written while the first author held NSF Grant r.P8019.

**Present address University of Maryland, College Park, Md. 30740.

${ }^{1}$ Figures in brackets indicate the literature references at the end of this paper. 
Going over to the representation of $\Gamma$ as $\operatorname{LF}(2, Z)$, we define the principal congruence subgroup $\Gamma(n)$ as the totality of elements $\left(\begin{array}{ll}a & b \\ c & d\end{array}\right) \in \Gamma$ such that

$$
a \equiv d \equiv \pm 1 \bmod n, \quad b \equiv c \equiv 0 \bmod n .
$$

\section{The Results}

We now state the principal results obtained. Throughout this section $G$ is a normal subgroup of $\Gamma$ of index $\mu$, level $n$, genus $g$, and having $t$ parabolic classes.

(1) Suppose that $\mu$ is square-free. Then either $G=\Gamma, \Gamma^{2}, \Gamma^{3}$, or $\Gamma(2)$, or else $G$ is of genus 1 and every prime divisor of $\mu / 6$ is $\equiv 1 \bmod 3$.

(2) Define $f(\mu)$ as 1 if there is a normal subgroup $G$ of index $\mu$ with solvable quotient group $\Gamma / G$, and 0 otherwise. Then

$$
\lim _{x \rightarrow \infty} \frac{1}{x} \sum_{\mu \leq x} f(\mu)=0
$$

(3) Let $p$ be a prime, $p \equiv-1 \bmod 3$, and suppose that $p>r$. Then there is no normal subgroup of $\Gamma$ of index $p r$.

(4) If $\Gamma / G$ is nilpotent then it is abelian, and $G$ must be $\Gamma, \Gamma^{2}, \Gamma^{3}$, or $\Gamma^{\prime}$.

(5) Let $p$ be a prime, $p \equiv 1 \bmod 12$. Then there are no normal subgroups of $\Gamma$ having $2 p$ parabolic classes.

(6) Let $p$ be a prime $>84, p \equiv-1 \bmod 3$; and let $n$ be any positive integer. Then there are no normal subgroups of $\Gamma$ of genus $1+p^{n}$.

(7) Let $p$ be a prime $>5$, and suppose that $\mu=6 p^{2}$. Then $G$ must be one of the following groups: groups:

(i) $\Gamma(2)^{p} \Gamma(2)^{\prime}$.

(ii) $(p, 0,1)$.

(iii) $\left(1, m_{1}, p^{2}\right),\left(1, m_{2}, p^{2}\right)$, where $p \equiv 1 \bmod 3$ and $m_{1}, m_{2}$ are the solutions of $m^{2}+m+1 \equiv 0$ $\bmod p^{2}$.

Thus

$$
N\left(6 p^{2}\right)=3+(p / 3), \quad p \text { prime, } p>5 .
$$

(8) Let $p$ be a prime $>11$. Then $N\left(12 p^{2}\right)=0$.

(9) Let $p$ be a prime $>11$. Then the only normal subgroup of $\Gamma$ of index $12 p^{3}$ is $\Gamma(3)^{p} \Gamma(3)^{\prime}$.

(10) $N(72)=2, N(78)=2, N(84)=0, N(90)=0$.

(11) There is just one normal subgroup of $\Gamma$ of genus 2: namely $G_{4,2}$.

(12) The normal subgroups of $\Gamma$ with $t$ parabolic clasese, $t \leqslant 5$, are the following:

$$
\begin{aligned}
& t=1: \Gamma, \Gamma^{2}, \Gamma^{3}, \Gamma^{\prime} . \\
& t=2: \text { none. } \\
& t=3: \Gamma(2),(1,1,3) . \\
& t=4:(2,0,1), \Gamma(3), G_{3,4} . \\
& t=5: \text { none. }
\end{aligned}
$$

\section{Some Remarks}

Perhaps the most striking results are the first two. A generalization of (2) with a precise estimate for the density function is in course of publication ([2]). As for (1), we note that if $G$ is any 
finite group of square-free order generated by elements $x, y$ such that $x^{2}=y^{3}=1$, then $(x y)^{6}=1$. This is so since the second commutator subgroup $G^{\prime \prime}$ is necessarily $\{1\}$ (p. 148 of [3]), and

$$
\begin{gathered}
a=x y x y^{2} \epsilon G^{\prime}, \quad b=x y^{2} x y \epsilon G^{\prime}, \\
(x y)^{6}=a b^{-1} a^{-1} b \epsilon G^{\prime \prime} .
\end{gathered}
$$

The result (1) is now an easy consequence.

The other results are of varying degrees of difficulty, but generally present no special problems.

\section{References}

[1] Greenberg, Leon, Note on normal subgroups of the modular group, Proc. Amer. Math. Soc. 17, 1195-1198 (1966).

[2] Greenberg, Leon, and Newman, Morris, Some results on solvable groups, Arch. Math. (to appear).

[3] Hall, Marshall, The Theory of Groups (The Macmillan Company, New York, 1959).

[4] Newman, Morris, Classification of normal subgroups of the modular group, Trans. Amer. Math. Soc. 126, 267-277 (1967).

[5] Newman, Morris, A complete description of the normal subgroups of genus one of the modular group, Amer. J. Math. 86, 17-24 (1964).

(Paper 74B2-324) 